\title{
Simposio
}

\section{CARCINOMA GÁSTRICO}

\author{
Gastric Carcinoma
}

\section{Carcinoma Gástrico: Factores de riesgo. Rol del Helicobacter pylori}

\author{
Gastric Carcinoma: Risk factors. \\ Role of Helicobacter pylori
}

Manuel Valdivieso Rodríguez, M.D. ${ }^{1}$

\section{Resumen}

El carcinoma gástrico es uno de los cánceres más frecuentes y más letales en el Perú. Los factores de riesgo son múltiples; destacando al sexo masculino y edad avanzada, ingesta alta de sal, alimentos conservados en sal, carnes y pescados ahumados o secos, alimentos encurtidos, baja refrigeración, baja ingesta de frutas y verduras con baja ingesta de vitaminas A y C, infecciones al virus Epstein Barr y al Helicobacter pylori, exposición a radiación, factores genéticos múltiples, bajos niveles de actividad física, obesidad y pobreza o bajo nivel socioeconómico. La infección de la mucosa gástrica por Helicobacter pylori, Carcinógeno tipo 1, produce lesiones progresivas y sucesivas que pueden evolucionar a través de una serie de pasos intermedios, de gastritis a cáncer. El daño de la infección en la mucosa gástrica puede ser reversible con el tratamiento de la infección excepto cuando se llega a metaplasia intestinal que con frecuencia representa el denominado "punto de no retorno". La emergencia de resistencia antibiótica del Helicobacter pylori impide la recuperación de las lesiones histológicas y facilita el desarrollo del cáncer gástrico.

Palabras clave: Cáncer gástrico, factores de riesgo, Helicobacter pylori.

\section{Abtract}

Gastric carcinoma is one of the most frequent and most lethal cancers in Peru. The risk factors are multiple; highlighting the male sex and advanced age, high salt intake, foods preserved in salt, smoked or dried meats and fish, pickled foods, low refrigeration, low intake of fruits and vegetables with low intake of vitamins A and C, Epstein Barr virus and Helicobacter pylori infections, radiation exposure, multiple genetic factors, low levels of physical activity, obesity, and poverty or low socioeconomic status. The infection of the gastric mucosa by Helicobacter pylori, a type 1 carcinogen, produces progressive and successive lesions that could evolve through a series of intermediate steps, from gastritis to cancer. The damage of the infection in the gastric mucosa can be reversible with the treatment of the infection except when intestinal metaplasia is reached, which often represents the so-called "point of no return". The emergence of antibiotic resistance of Helicobacter pylori prevents the recovery of histological lesions and facilitates the development of gastric cancer.

Keywords: Gastric cancer, riskfactors, Helicobacter pylori. 


\section{Antecedentes}

El cáncer es un mal frecuente y se estima que en el año 2020, hubieron más de 19 millones de diagnósticos nuevos y más de 9 millones de muertes debidas al cáncer en el mundo (Tabla 1). En orden descendiente, los cánceres más frecuentes son los de mama, pulmón, colorectal, próstata, estómago, hígado y cuello uterino. La mortalidad varía de cáncer a cáncer y en forma descendiente las causas de muerte más frecuentes por cáncer en el mundo son los de pulmón, colorectal, hígado, estómago, mama, esófago y cuello uterino ${ }^{(1)}$.

\begin{tabular}{|c|c|c|c|}
\hline \multicolumn{4}{|c|}{ Tabla 1} \\
\hline \multicolumn{2}{|c|}{ INCIDENCIA } & \multicolumn{2}{|c|}{ MORTALIDAD } \\
\hline NOMBRE & NÚMERO & NOMBRE & NÚMERO \\
\hline Mama & 2'261,419 & Pulmón & $1^{\prime} 796,144$ \\
\hline Pulmón & $2 ' 206,771$ & Colorectal & 915,880 \\
\hline Colorectal & 1'931,590 & Hígado & 830,180 \\
\hline Próstata & $1 ' 414,259$ & Estómago & 768,793 \\
\hline Estómago & $1 ' 089,103$ & Mama & 684,996 \\
\hline Hígado & 905,677 & Esófago & 544,076 \\
\hline Cuello uterino & 604,127 & Cuello uterino & 341,831 \\
\hline Total & $19 ' 292,789$ & Total & 9'958,133 \\
\hline
\end{tabular}

El carcinoma del estómago es uno de los más frecuentes, ocupando el quinto lugar en frecuencia, y el número cuatro en mortalidad global en el año 2020. Esto representa 1'089,103 de diagnósticos nuevos y 768,793 de muertes al año. Es más común en el Asia Oriental, particularmente en China, Japón, Corea del Sur; Europa del Este, Centroamérica y Sudamérica. Áreas de baja incidencia incluyen a Australia, Nueva Zelanda, América del Norte, Europa Occidental, Asia -sub Central y la mayor parte del África.

La frecuencia de casos nuevos y de mortalidad debida al cáncer en forma descendiente en el Perú se muestra en la tabla 2. De acuerdo a Globocan en el año 2020, el cáncer gástrico fue tercero en frecuencia detrás solamente a cánceres de próstata y mama; mientras que fue el primero en mortalidad seguido de cánceres del pulmón, próstata y colorectal ${ }^{(1)}$. La mortalidad por el cáncer de estómago en nuestro medio y en muchos países de Centroamérica y América del Sur se debe a lo avanzado de este cáncer cuando se le diagnostica. Se estima que hasta el 75\% de pacientes con cáncer, incluyendo los del estómago, se

\begin{tabular}{|llll|}
\multicolumn{4}{c}{ Tabla 2} \\
Incidencia y mortalidad por el cáncer \\
en el Perú en forma descendiente
\end{tabular}

presentan al Instituto Nacional de Enfermedades Neoplásicas de Lima en estado avanzado ${ }^{(2)}$.

\section{Factores de riesgo}

Los factores de riesgo para el cáncer gástrico son múltiples (Tabla 3). Incluyen al sexo masculino, edad avanzada, ingesta alta de sal, alimentos conservados en sal, carnes y pescados ahumados o secos, alimentos encurtidos, baja refrigeración, baja ingesta de frutas y verduras con baja ingesta de vitaminas A y C; infección debida al Helicobacter pylori y al virus Epstein Barr, exposición a radiación, factores genéticos múltiples, bajos niveles de actividad física, obesidad y nivel socioeconómico bajo ${ }^{(3-5)}$.

\begin{tabular}{|cc|}
\hline \multicolumn{2}{|c|}{ Tabla 3} \\
\hline Factores de riesgo para el carcinoma gástrico \\
\hline •Edad y sexo masculino & $\bullet$ Helicobacter pylori \\
\hline -Factores nutricionales & $\bullet$ Virus Epstein - Bar \\
- Dieta alta en sal & $\bullet$ Exposición a radiación \\
- Dieta alta en nitritos & •Factores genéticos \\
- Falta de refrigeración & - Grupo sanguíneo A \\
- Baja vitaminas A y C & -Anemia perniciosa \\
\hline -Ocupacional & -Historia familiar \\
- Trabajadores del jebe & -HNPCC \\
- Trabajadores de minas & -Sindrome de Li - Fraumeni \\
\hline Fumadores de tabaco & $\bullet$ Lesiones pre-cancerosas \\
\hline
\end{tabular}


La relativa importancia de diferentes factores de riesgo en relación al desarrollo del carcinoma gástrico es variable y difícil de especificar. La falta de refrigeradora en, por lo menos, el $51 \%$ de hogares del Perú es preocupante ya que ello conlleva un mal estado de conservación de alimentos en casa ${ }^{(6)}$. Se dice que la falta de ingesta de frutas y de vegetales en la dieta son responsables por el $40 \%$ al $30 \%$ de cánceres gástricos. El tabaquismo e infecciones por el virus Epstein Barr son importantes en el $18 \%$ y $13 \%$ respectivamente. Algunos proveen la información sobre el riesgo de desarrollar cáncer gástrico como número de veces que este ocurriría si la infección por el Helicobacter pylori está presente ( 2 a 20 veces), la presencia de gastritis atrófica (3 a 18 veces), de anemia perniciosa ( 2 a 6 veces) o la presencia de bajo estado socioeconómico $(2 \text { veces })^{(7-9)}$.

La distribución de la pobreza y de la mortalidad por carcinoma gástrico en los diferentes Departamentos del Perú fue mostrada por Piñeros et al. ${ }^{(10)}$ (Figuras 1 y 2). En el panel izquierdo de la figura 1 se ven los diferentes departamentos de la costa, la sierra y de la selva. En el panel de la derecha de esta figura se observa la severidad porcentual de la pobreza reflejada por la intensidad de la coloración roja/morada que es mayor en ciertas partes de la sierra y de la selva. La pobreza es más alta en los departamentos de Huancavelica, Ayacucho y
Apurimac; pero también en Cajamarca, Huánuco, Pasco, Piura y Puno. La figura 2 muestra que el porcentaje de mortalidad por cáncer gástrico es más alto también en regiones pobres de la sierra del Perú como son Huánuco, Huancavelica, Pasco y Cajamarca siendo la mortalidad en estas regiones de dos a tres veces más alta que la cantidad promedio de mortalidad del cáncer gástrico de 16.3/100,000 habitantes. Por consiguiente, la incidencia y la mortalidad por cáncer del estómago en el Perú son más altas en regiones pobres de la sierra. Esta observación de mayor incidencia y de mortalidad por cáncer gástrico en las regiones montañosas de América Latina ha sido reportada por otros autores anteriormente ${ }^{(11)}$.

\section{Rol del Helicobacter pylori}

Uno de los factores de riesgo más importantes en cáncer gástrico es la infección por el Helicobacter pylori ${ }^{(12)}$. Este organismo flagelar gran negativo fue identificado como causa de úlceras del estómago por Marshall y Warren en 1983 y ello los llevó a recibir el premio Nobel de Fisiología en 2005. El Helicobacter pylori está presente en un $50 \%$ de la población del mundo siendo mucho más común en áreas donde existe más casos de cáncer del estómago. El Helicobacter pylori tiene potencial carcinogénico de fuerza y se le conoce como

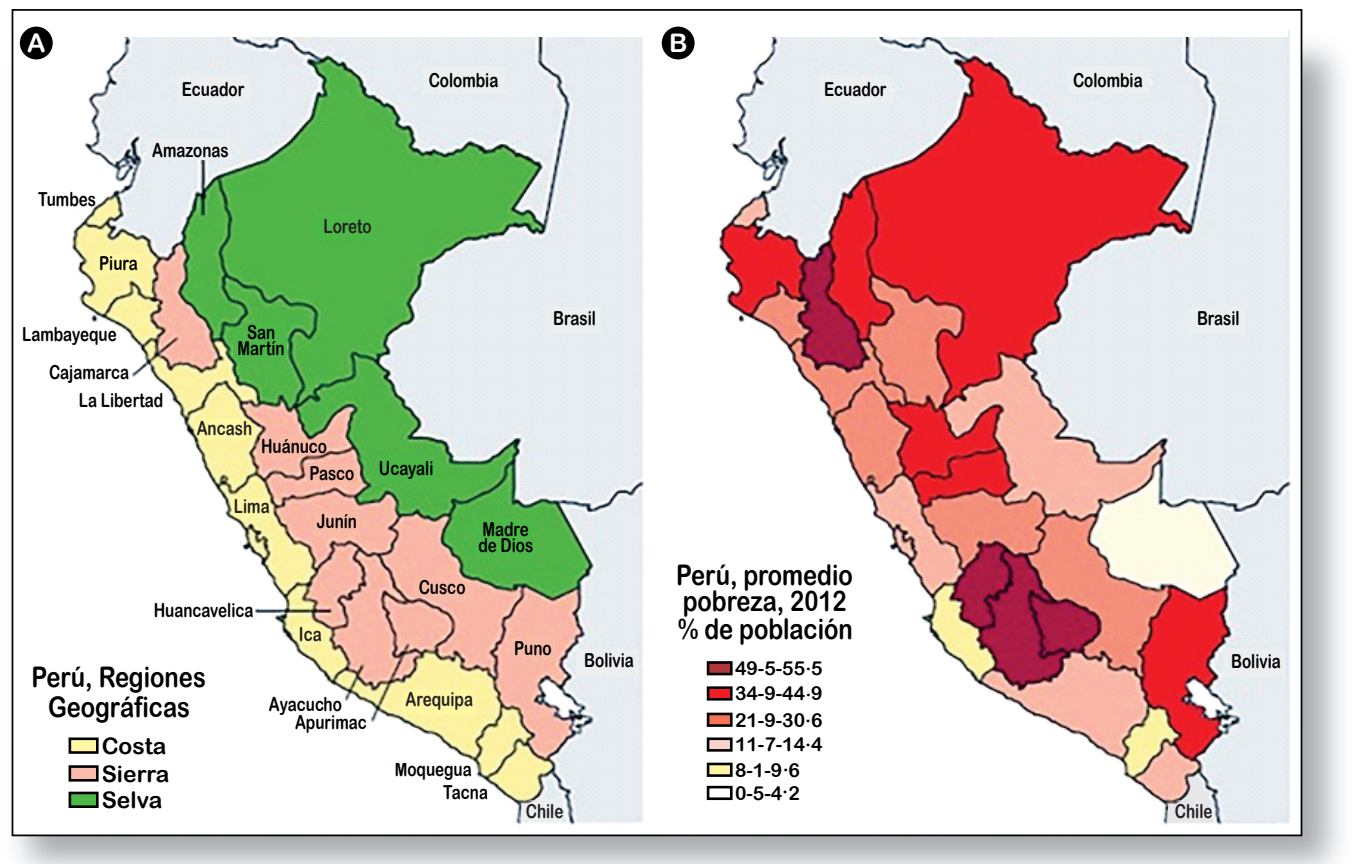

Figura 1. El Estado de Pobreza en el Perú de acuerdo a Departamento. La Figura muestra los Departamentos de la costa, sierra y selva del Perú en el lado izquierdo y, el grado de pobreza en estos Departamentos de acuerdo a la intensidad del color rojo/morado en el lado derecho. De: Marion Piñeros et al. Lancet Oncol 2017;18 e573-586. 


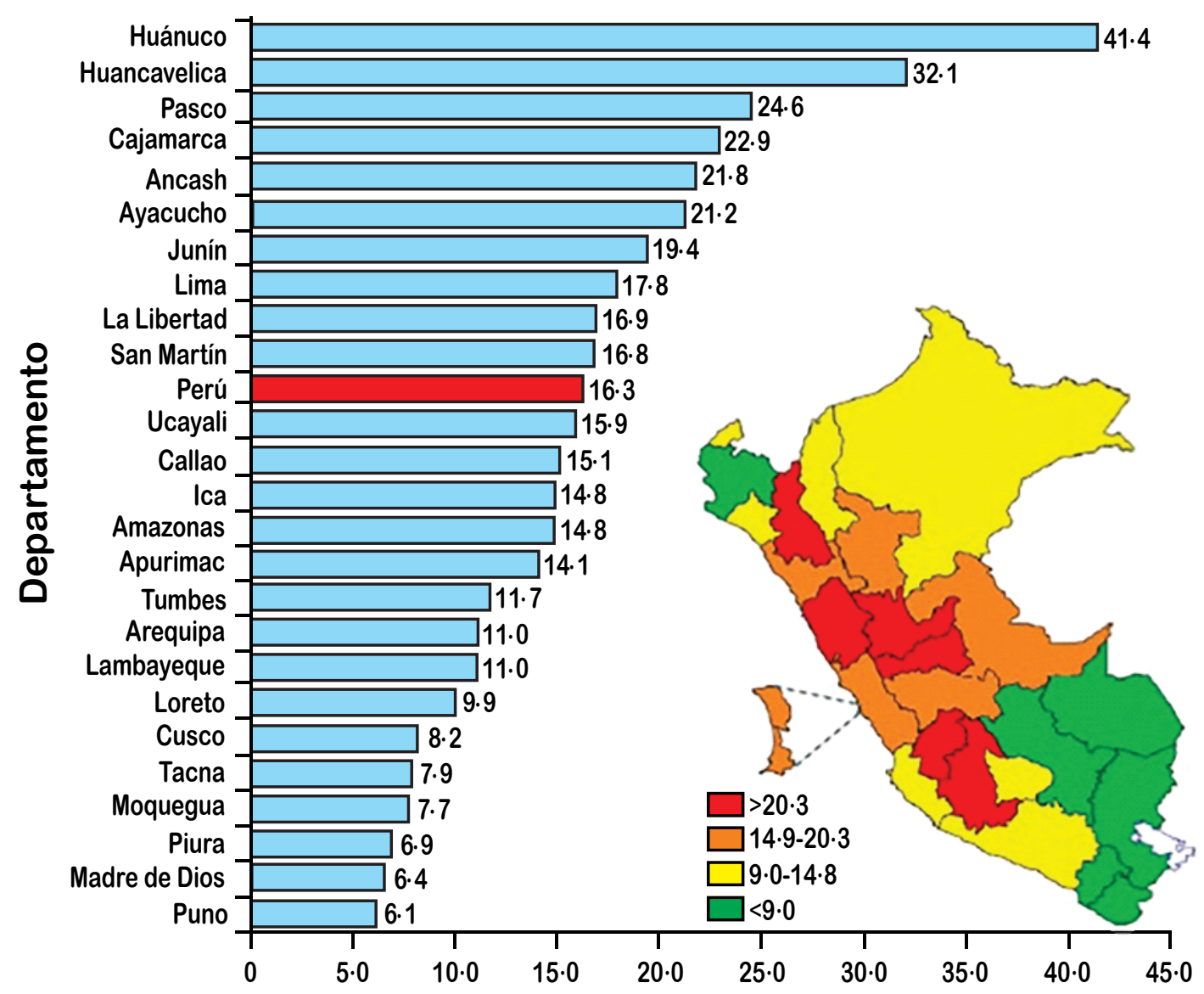

Figura 2: Mortalidad por Cáncer Gástrico en el Perú de acuerdo a Departamento. La figura muestra que la mortalidad por Carcinoma Gástrico es más alta en los Departamentos más pobres de la sierra como Huánuco, Huancavelica y Pasco. De: Marion Piñeros et al. Lancet Oncol 2017;18 e573-586.

Carcinógeno de Tipo I, como lo es el cigarrillo en relación al cáncer del pulmón. El Helicobacter pylori produce una serie de lesiones consecutivas en la mucosa gástrica, conocida como la "Cascada de Correa" en homenaje al Dr. Pelayo Correa, quien describió esta secuencia ${ }^{(12,13)}$. El Helicobacter pylori no es compatible con el medio ácido del estómago pero sobrevive en él debido a la presencia de catalasa y ureasa que neutralizan al acido gástrico.

La infección por el Helicobacter pylori es más frecuente en medios socioeconómicos bajos y es generalmente asintomática. De acuerdo a Peek et al. ${ }^{(14)}$, el Helicobacter pylori causa de por si una serie de trastornos que incluyen la úlcera péptica en el 10\% de casos, adenocarcinoma gástrico en un 2$3 \%$ y en menor frecuencia el linfoma de la mucosa gástrica o Maltoma en un $<0.1 \%$. La irritación crónica causada por la infección al Helicobacter pylori estimula la generación de radicales de oxígeno y de nitrógeno que son dañinos para el
ADN, causando mutaciones en las células madres del estómago (14). El factor de patogenicidad Cag A del Helicobacter pylori produce alteraciones en la metilación del ADN y degrada e inactiva al gene protector $\mathrm{p} 53^{(15)}$. Debe notarse que la presencia del factor de virulencia Cag A no es constante estando presente en un $100 \%$ de las sepas de Helicobacter pylori en el Asia y en un $60 \%$ de las sepas del Occidente ${ }^{(14)}$.

La secuencia de lesiones gástricas causadas por el Helicobacter pylori incluyen la gastritis crónica, la metaplasia intestinal, la displasia y el cáncer. La gastritis crónica representa un estado de inflamación crónica que causa hipoclorhidria, la cual altera el metabolismo del fierro y se asocia con alteraciones del microbioma intestinal ${ }^{(14-16)}$. La gastritis crónica aumenta 10 veces la posibilidad de desarrollar cáncer gástrico. Antes de llegar al siguiente paso, metaplasia intestinal, el tratamiento de la infección causada por el Helicobacter pylori puede mejorar el daño histológico de la 
mucosa del estómago. En metaplasia intestinal es más difícil de hacerlo. Por ello, la metaplasia intestinal es llamada el "punto de no retorno"(17). Estudios en Corea del Sur han demostrado que este estadio se asocia con una posibilidad alta de 187 veces de desarrollar cáncer gástrico en comparación a controles. Además, el tiempo medio del desarrollo del cáncer es de 6.1 años ${ }^{(17)}$. El siguiente estadio es el de displasia en la que Huang et al. han descrito amplificaciones del cromosoma 8q que consiste en la duplicación de parte del brazo largo del cromosoma 8 y de tener telómeros $\operatorname{cortos}^{(18)}$. Los telómeros son una secuencia del ADN que están en los extremos del cromosoma protegiendo la integridad del ADN. La agresividad de este estadio se aprecia en el desarrollo del cáncer en solo 2.6 años ${ }^{(17)}$.

El progreso de lesiones gástricas sospechadas debidas a infección por Helicobacter pylori se aprecia también en países con incidencia baja de este cáncer (Tabla 4). En el estudio de Song et al. en Suecia se evaluaron 405,172 pacientes con lesiones gástricas entre 1979 y $2011^{(19)}$. Se demostró que la evolución progresiva de lesiones del estómago se asoció con un incremento en la frecuencia del carcinoma gástrico de 1 en pacientes con mucosa normal hasta 6.5 veces en pacientes con displasia. Este riesgo progresivo de desarrollar cáncer gástrico con el avance de las lesiones de la mucosa del estómago también se apreció viendo la evaluación porcentual del desarrollo del cáncer de 1 en 256 personas con mucosa normal a 1 en 19 personas cuando los pacientes tuvieron displasia. Por consiguiente, existe una relación linear entre la severidad de las lesiones gástricas causadas por el Helicobacter pylori y la posibilidad de desarrollar cáncer gástrico aún en países con baja incidencia de cáncer gástrico.

\begin{tabular}{|lcc|}
\hline \multicolumn{3}{|c|}{ Tabla 4} \\
\hline \multicolumn{2}{|c|}{ Injcidencia cumulativa de cáncer en Suecia de acuerdo a } \\
lesiones de la mucosa gástrica: & 405,172 pacientes (1979-2011) \\
\hline RIESGO DE CÁNCER & RIESGO DE CÁNCER A VEINTE AÑOS \\
Mucosa normal & 1 & 1 en 256 personas \\
Gastritis & 1.8 & 1 en 85 personas \\
Gastritis atrófica & 2.8 & 1 en 50 personas \\
Metaplasia intestinal & 3.4 & 1 en 39 personas \\
Displasia & 6.5 & 1 en 19 personas \\
\hline
\end{tabular}

El rol del Helicobacter pylori como agente causal del cáncer gástrico es variable a través del mundo. El estudio de Pilamides et al. ${ }^{(20)}$ examinó esta variabilidad en el África, donde el riesgo de carcinoma gástrico es bajo a pesar de tener infecciones causadas por el Helicobacter pylori. El estudio comparó la patogenicidad de la infección en Nigeria y en Sud África y se apreció, que a pesar de que en ambas regiones existe la infección por el Helicobacter pylori, que el porcentaje de erosiones, ulceraciones y de cáncer gástrico debido al Helicobacter pylori fueron más altos en Nigeria que en Sud África. El estudio de Pilamides en Nigeria y Sud África incluyó endoscopias y biopsias gástricas en ambas regiones para aislar al Helicobacter pylori y estudiar sus diferentes marcadores moleculares en relación a los hallazgos patológicos. Encontraron que la composición molecular del Helicobacter pylori desde el punto de vista de la expresión de los factores patogenéticos Cag A y Vac A fue diferente y mucho más frecuente en Nigeria que en Sud África. Igualmente, se observó que la composición genética del Vac A fue diferente constituyendo un mosaicismo distinto entre Nigeria y Sud África. Por consiguiente, parecería que el diferente grado de lesiones gástricas causadas por el Helicobacter pylori entre Nigeria y Sud África se debe en parte a diferentes expresiones de los factores de patogenicidad de sus marcadores moleculares Cag A y Vac A. Esta observación de tener infecciones por el Helicobacter pylori y poco cáncer gástrico con grados diferentes de expresión de los factores patogenéticos Cag A y Vac A es representativa del llamado "Enigma Africano"(Tabla 5).

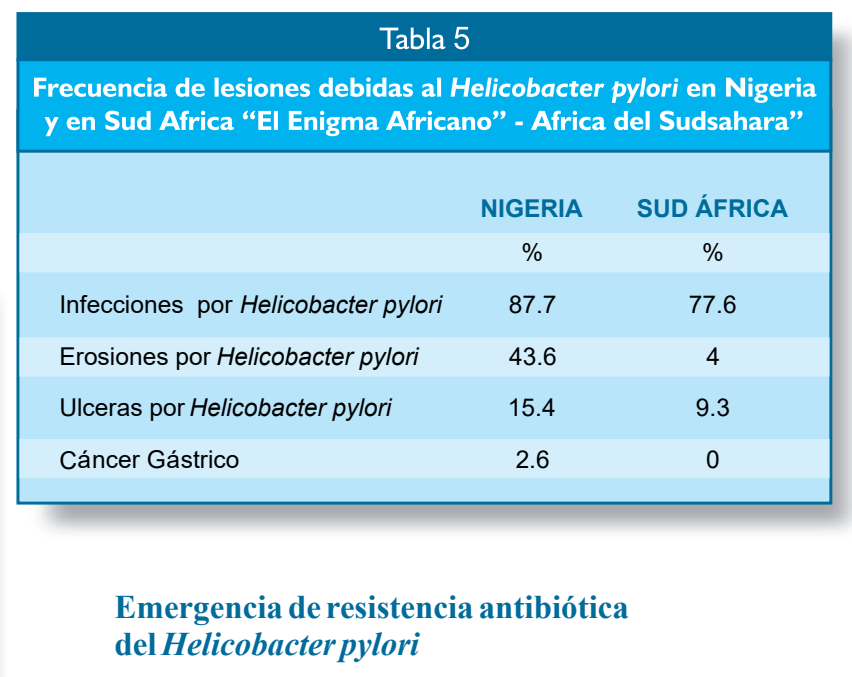

Otros puntos importantes en la evaluación de los factores de riesgo para el carcinoma gástrico en pacientes infectados por el Helicobacter pylori están basados en fuentes ambientales de contaminación que facilitan la infección y el desarrollo de resistencia antibiótica del Helicobacter. En una colaboración entre las Universidades de Michigan en Ann Arbor, Cayetano Heredia en Lima y la División de Salud 
Ambiental del MINSA en Lima, se estudió la epidemiologia molecular del Helicobacter pylori con la hipótesis de que el agua potable de Lima era una posible fuente de contaminación de la infección ${ }^{(21)}$. Aunque no se pudo cultivar la presencia del Helicobacter pylori en el agua potable, se demostró la presencia qualitativa y quantitativa del ADN especifico del Helicobacter pylori en el agua de la cocina de pacientes infectados con esta bacteria. Además, se vio que la respuesta al tratamiento triple con Amoxicilina, Claritromicina e Isomeprazol fue de solo $66 \%$ que estuvo muy debajo de lo esperado en América Latina ${ }^{(22)}$. El seguimiento de estos pacientes y el tratamiento con terapia de segunda línea (tetraciclina, furazolidona, subsalicilato de bismuto y pantoprazol) a aquellos que no respondieron al tratamiento inicial, elevaron la respuesta al $85 \%$ al año del estudio.

Se sospechó que la baja respuesta al tratamiento fue debida a la presencia de resistencia antibiótica, la que fue demostrada en la evaluación de 76 sepas del Helicobacter pylori de pacientes del estudio ${ }^{(23)}$. Usando el método europeo del EUCAST ${ }^{(24)}$, se demostró resistencia antibiótica entre el $33 \%$ y el $62 \%$ de sepas a Amoxicilina, Claritromicina, Levofloxacino y Mitronidazol, que son drogas de primera línea para el tratamiento de infección por el Helicobacter pylori. Esta resistencia antibiótica del Helicobacter pylori ha sido descrita en diferentes regiones del mundo y como ejemplo, se cita la revisión de Vu Van Khien et al. en Vietnam quien notó seis estudios incluyendo a 2,318 sepas del Helicobacter pylori, demostrando resistencia antibiótica a Amoxicilina, Claritromicina, Metronidazol y Levofloxacino en el 15\%, 34\%, 69\% y $27 \%$ de sepas respectivamente ${ }^{(25)}$ (Tabla 6).

\section{Conclusión}

Los factores de riesgo para el cáncer gástrico son múltiples. Incluyen al sexo masculino y edad avanzada, ingesta alta de sal, alimentos conservados en sal, carnes y pescados ahumados o secos, alimentos encurtidos, baja refrigeración, baja ingesta de frutas y verduras con baja ingesta de vitaminas A y C; infección al Helicobacter pylori, exposición a radiación, factores genéticos múltiples, bajos niveles de actividad física, obesidad y pobreza o bajo nivel socioeconómico.

Uno de los factores de riesgo más importante es la presencia de infección por el Helicobacter pylori. Se le considera Carcinógeno tipo 1 debido a su efecto carcinogénico directo en la mucosa gástrica con lesiones progresivas que constituyen la

\begin{tabular}{|cccc|}
\hline \multicolumn{4}{c|}{ Tabla 6} \\
\hline \multicolumn{4}{c|}{ Resistencia antibiótica de 76 sepas clínicas de } \\
Helicobacter pylori (EUCAST) \\
\hline & $>0.125$ & 25 & 33 \\
Amoxicilina & 0.5 & 27 & 36 \\
Claritromicina & $>1$ & 3 & 4 \\
Tetraciclina & $>1$ & 41 & 54 \\
Levofloxacino & $>8$ & 47 & 62 \\
Metronidazol & $>1$ & 35 & 46 \\
Rifampicina & &
\end{tabular}

"Cascada de Correa". La respuesta a la inflamación crónica y sostenida causada por el Helicobacter produce lesiones que pueden evolucionar, a través de una serie de pasos intermedios, de gastritis a cáncer. El daño de la infección en la mucosa gástrica puede ser reversible con el tratamiento de la infección excepto cuando se llega a la fase de metaplasia intestinal que con frecuencia representa el denominado "punto de no retorno". El desarrollo de resistencia antibiótica del Helicobacter pylori es preocupante ya que impide la recuperación de las lesiones histológicas al tratamiento del Helicobacter pylori y facilita así el desarrollo del cáncer gástrico.

Hacemos un llamado a nuestras autoridades políticas y de salud pública para elaborar medidas de prevención del carcinoma gástrico que lleve a disminuir los factores de riesgo arriba mencionados, por lo menos en nuestra región andina donde las condiciones de salubridad y el estado de pobreza de su población incrementan el riesgo de desarrollar cáncer gástrico. Por otro lado, ya pasó el momento en el que las sociedades científicas médicas y el Ministerio de Salud del Perú desarrollen políticas de detección temprana de lesiones gástricas pre-cancerosas para su monitoreo y seguimiento uniforme a través del país y así llegar a la detección temprana del cáncer gástrico tal como se hace en el Japón y en Corea del Sur. En estos países, personas mayores de 40 años son candidatos para endoscopia del estómago, las que se repiten cada uno o dos años dependiendo del tipo de lesión premaligna presente. Con este sistema de monitoreo endoscópico se puede detectar carcinoma gástrico en estadios tempranos donde su resección conlleva una curación de más del 80 - 90\%. Este tipo de política sanitaria debe ir mano a mano con programas de seguro de salud que cubrirían los exámenes de detección con gastroscopias preventivas y diagnósticas. Solo así podríamos mejorar el diagnóstico y el pronóstico de este terrible cáncer. 
Referencias bibliográficas

1.International Agency for Research on Cancer; World Health Organization; GLOBOCAN 2020.

2.Salazar MR, Regalado-Rafael R, Navarro JM, Montanez DM, Abugattas JE, Vidaurre T. El Instituto Nacional de Enfermedades Neoplásicas en el control del cáncer en el Perú. Rev Peru Med Exp Salud Pública. 2013;30(1):105-112.

3.Jaw-Town Lin. Screening of Gastric Cancer: Who, When, and How. Clinical Gastroenterology and Hepatology 2014;12:135138.

4. Crew KD, Neugut AI. Epidemiology of gastric cancer. World J Gastroenterol 2006;12:354-362.

5. Fock KM, Talley N, Moayyedi P, Hunt R, Azuma T, Sugano K. et al. Asia-Pacific consensus guidelines on gastric cancer prevention. J Gastroenterol Hepatol 2008;23:351-365.

6.Perú: Perfil Sociodemográfico 2017. Instituto Nacional de Estadística e Informática. Censos Nacionales de Población y Vivienda 1993, 2007 y 2017. Capítulo 6: Característica de los hogares; páginas 354-391.

7.de Vries AC, Haringsma J, Kuipers EJ. The detection, surveillance and treatment of premalignant gastric lesions related to Helicobacter pylori infection. Helicobacter 2007; $12: 1-15$

8. de Vries AC, van Grieken NC, Looman CW, Casparie MK, de Vries E, Meijer GA, et al. Gastric cancer risk in patients with premalignant gastric lesions: a nationwide cohort study in the Netherlands. Gastroenterology 2008;134:945-952.

9. On On Chan A, Wong B. Risk Factors for Gastric Cancer. UpToDate 2020. Literature review current through: Dec 2020.

10. Piñeros M, Ramos W, Antoni S, Abriata G, Medina LE, Miranda JJ, et al. Cancer in Peru 1. Cancer patterns, trends, and transitions in Peru: a regional perspective. thelancet.com/ oncology 2017; 18:e573-e586.

11. Torres J, Correa P, Ferreccio C, Hernandez-Suarez G, Herrero R, Cavazza-Porro M, et al. Gastric cancer incidence and mortality is associated with altitude in the mountainous regions of Pacific Latin America. Cancer Causes Control. 2013;24(2):249-256

12. Correa P, Haenszel W, Cuello C, Tannenbaum S, Archer M. A model for gastric cancer epidemiology. Lancet 1975;2:58-60.

13. Correa P, Piazuelo MB. The gastric precancerous cascade. J Dig Dis. 2012;13(1):2-9.

14. Peek RM, Crabtree JE. Helicobacter infection and gastric neoplasia. J Pathol 2006;208 (2):233-248.

15. Buti L, Spooner E, Van der Veen AG, Rappuoli R, Covacci A, Ploeghet HL. Helicobacter pylori cytotoxin-associated gene A (CagA) subverts the apoptosis-stimulating protein of p53 (ASPP2) tumor suppressor pathway of the host. Proc Natl Acad Sci U S A 2011;108:9238-9243.
16.Guo Y, Zhang Y, Gerhard M, Gao JJ, Mejias-Luque R, Zhang L, et al. Effect of Helicobacter pylori on gastrointestinal microbiota: a population-based study in Linqu, a high-risk area of gastric cancer. Gut 2019;0:1-10. doi:10.1136/gutjnl2019-319696

17. Koulis A, Buckle A, Boussioutas A. Premalignant lesions and gastric cancer: Current understanding. World J Gastrointest Oncol 2019;11(9):665-678.

18.Huang KK, Ramnarayanan K, Zhu F, Srivastava S, Xu C, Tan ALK, et al. Genomic and Epigenomic Profiling of HighRisk Intestinal Metaplasia Reveals Molecular Determinants of Progression to Gastric Cancer. Huang et al., Cancer Cell. 2018;33:137-150.

19.Song H, Ekheden IG, Zheng Z, Ericsson J, Nyrén O, Ye W. Incidence of gastric cancer among patients with gastric precancerous lesions: observational cohort study in a low risk Western population. BMJ 2015;351:h3867 doi: 10.1136/bmj.h 3867.

20.Pilamides P, Jolaiya T, Idowu A, Loell E, Onyekwere C, Ugiagbe $\mathbf{R}$, et al. Helicobacter pylori patient isolates from South Africa and Nigeria differ in virulence factor pathogenicity profile and associated gastric disease outcome. Scientific Reports; Nature Research. 2020;10:11409. https://doi.org/10.1038/s41598-020-66128-0

21. Valdivieso, M, Bussalleu, A, Sexton, R, Boehnke, K, Osorio, S, Novoa I, et al. Clinical, Epidemiologic, and Genomic Studies (SWOG S1119) of Helicobacter Pylori in Lima, Peru: Role of Contaminated Water. J. of Cancerol. 2016;2:52-63.

22.Greenberg ER, Anderson GL, Morgan DR, Torres J, Chey WD, Bravo LE, et al. 14-day triple, 5-day concomitant and 10day sequential therapies for Helicobacter pylori infection in seven Latin American sites: a randomized trial. Published on line by The Lancet on July 20, 2011. DOI: 10.1016/S0140-6736 (11) 60825-8.SWOG.

23.Boehnke K, Valdivieso M, Bussalleu A, Sexton R, Thompson KC, Osorio S, et al. Antibiotic resistance among Helicobacter pylori clinical isolates in Lima, Peru (SWOG Clinical Trial 1119). Infection and Drug Resistance; 2017:10:85-90.

24.European Committee on Antimicrobial Susceptibility Testing Breakpoint tables for interpretation of MICs and zone diameters. Breakpoint tables for interpretation of MICs and zone diameters. Version 5.0 [Internet]. Proteus. 2015. Available from: http://www.eucast.org

25.Khien VV, Thang DM, Hai TM, Duat NQ, Khanh PH, Ha DT, et al. Management of Antibiotic-Resistant Helicobacter pylori Infection: Perspectives from Vietnam. Gut and Liver. 2019;1(5);483-497.

Contribución de autoría: Manuel Valdivieso Rodríguez ha sido el autor de esta publicación, contribuyendo a su concepción, organización de la presentación científica, búsqueda electrónica, revisión inicial, redacción, revisión y aprobación final.

Conflicto de interés: El autor no tiene conflicto de interés con la publicación de este trabajo.

Financiamiento: Autofinanciado.

Citar como: Valdivieso Rodríguez M. Carcinoma Gástrico: Factores de riesgo. Rol de Helicobacter Pylori.

Diagnóstico(Lima). 2021;60(2):79-85.

DOI: 10.33734 /diagnostico.v60i2.284

Correspondencia: Manuel Valdivieso Rodríguez. Correo electrónico: m.valdivieso.md@outlook.com 